\title{
Histamine-induced end-tidal inspiratory activity and lung receptors in cats
}

\author{
N.E.L. Meessen*, C.P.M. van der Grinten*, H.Th.M. Folgering**, S.C.M. Luijendijk*
}

Histamine-induced end-tidal inspiratory activity and lung receptors in cats. N.E.L. Meessen, C.P.M. van der Grinten, H.Th.M. Folgering, S.C.M. Luijendijk. C)ERS Journals Ltd 1995.

ABSTRACT: Hyperinflation in acute asthma has been associated with inspiratory muscle activity, which persist during expiration. The main objective of the present study was to evaluate the role of rapidly adapting receptors (RARs), slowly adapting receptors (SARs) and C-fibre endings in generating end-tidal inspiratory activity (ETIA).

ETIA was induced by intravenous administration of histamine and continuous negative airway pressure (CNAP) in anaesthetized, spontaneously breathing cats. To differentiate between reflex activities from the three types of lung receptors, both vagus nerves were cooled to eight different temperatures $\left(T_{\mathrm{vg}}\right)$ between 4 and $37^{\circ} \mathrm{C}$. It is known that CNAP stimulates RARs and inhibits SARs. Histamine was used to stimulate RARs, and this was combined with continuous positive airway pressure (CPAP) to further stimulate SARs.

ETIA was evoked in the diaphragm and in parasternal intercostal muscles by both stimuli (histamine and CNAP) in 8 out of 18 cats. After vagotomy, neither histamine nor CNAP evoked ETIA any more. At $T_{\mathrm{vg}}=37^{\circ} \mathrm{C}$, CPAP suppressed histamineinduced ETIA; whereas, this suppression was diminished at $T_{\mathrm{vg}}$ between 14 and $8^{\circ} \mathrm{C}$. ETIA sharply declined for $T_{\mathrm{vg}}$ between $8^{\circ}$ and $4^{\circ} \mathrm{C}$, and at $T_{\mathrm{vg}}=4^{\circ} \mathrm{C}$ ETIA had virtually disappeared. At $\boldsymbol{T}_{\mathrm{vg}}=37^{\circ}$ and $22^{\circ} \mathrm{C}$ values of ETIA during CNAP were larger than those in response to histamine; whereas, at $T_{\mathrm{vg}}=10^{\circ} \mathrm{C}$ comparable ETIA values were obtained.

It was shown that ETIA is a vagal reflex activity in which C-fibre endings are not involved. Histamine-induced ETIA originates from stimulation of RARs, and is inhibited by stimulation of SARs. Mechanical stimulation of RARs is a forceful stimulus to induce ETIA. This suggests that hyperinflation in acute asthma might be due, at least in part, to ETIA resulting from an imbalance between SAR and RAR activity.

Eur Respir J., 1995, 8, 2094-2103.
*Dept of Pulmonology, University Hospital Maastricht, University of Limburg, The Netherlands. **Dept of Pulmonary Diseases Dekkerswald, University of Nijmegen, The Netherlands.

Correspondence: N.E.L. Meessen

Dept of Pulmonology

P.O. Box 5800

6202 AZ Maastricht

The Netherlands

Keywords:

continuous negative airway pressure

electromyogram

histamine

inspiratory muscle activity

pulmonary receptors

Received: June 151993

Accepted after revision July 241995

This study was supported by the Netherlands Asthma Foundation (NAF-88.26).
Bronchoconstriction and hyperinflation are characteristics of acute asthma [1]. Hyperinflation is considered to be disadvantageous to inspiratory muscle function, more so to the diaphragm than to the parasternal intercostal muscles (ICMs) [2]. Bronchoconstriction with an increased mechanical time constant and incomplete emptying of the lungs is one of the causes of hyperinflation [3]. Another factor is a persisting inspiratory muscle activity during expiration [4-6]. The electrical activity observed in the electromyogram (EMG) of inspiratory muscles at the end of expiration - just prior to the phasic increase in inspiratory activity - has been called tonic inspiratory activity $[4,6]$. As the origin of this so-called tonic activity is not yet clear, we prefer to use the term end-tidal inspiratory activity (ETIA). In asymptomatic asthmatics, it has been found that during histamine-induced hyperinflation the end-tidal pleural pressure was more negative than the predicted chest wall relaxation pressure at the corresponding end-tidal lung volume $[4,5]$, indicating the presence of ETIA. In more direct measurements, by evaluating electromyograms (EMGs) of inspiratory muscles, ETIA has been observed in human subjects after administration of histamine [6]. These studies have shown a significant correlation between the increase in thoracic gas volume and the increase in ETIA. Recent studies have shown that, with intact vagus nerves, lowering of tracheal pressure $\left(P_{\mathrm{tr}}\right)$ could evoke ETIA in rabbits [7] and cats [8, 9]. Stimulation of thin vagal afferents by administration of histamine increased the magnitude of ETIA in rabbits [10].

Although it has been indicated by several studies that histamine can induce ETIA, the mechanism is still uncertain. It has been shown that histamine stimulates 
rapidly adapting receptors (RARs) both by a direct chemical effect and by an indirect mechanical effect resulting from bronchoconstriction [11-14].

The main objective of the present study was to evaluate the role of RARs, slowly adapting receptors (SARs) and C-fibre endings, in histamine-induced ETIA. EMGs from the diaphragm and the ICMs were recorded during control conditions and after intravenous administration of histamine, both with and without simultaneous application of continuous positive airway pressure (CPAP). Although histamine and CPAP are not specific stimuli for one particular type of lung receptor, it is generally agreed that RARs are stimulated by histamine, whereas CPAP mainly activates SARs [15]. In order to further differentiate between the reflex activities of the lung receptors, the experiments were performed at different temperatures of the cervical vagus nerves $\left(T_{\mathrm{vg}}\right)$. To investigate the origin of ETIA, ETIA was also evoked by continuous negative airway pressure (CNAP), and its magnitude was compared to that of histamine-induced ETIA at various vagal temperatures. During CNAP, RARs are stimulated mechanically and SAR activity is inhibited. Effects on ETIA of both stimuli (histamine and CNAP) were also studied after vagotomy.

Preliminary data have been reported previously in abstract form [16].

\section{Methods}

\section{Animals and anaesthesia}

Experiments were performed on 18 adult cats of either sex, body weight $4.8 \pm 0.2 \mathrm{~kg}$ (mean \pm SEM). They were anaesthetized with ketamine-hydrochloride $\left(10 \mathrm{mg} \cdot \mathrm{kg}^{-1}\right.$ body weight, i.m.) and by intravenous injection of a chloralose-urethane mixture (12.5 and $62.5 \mathrm{mg} \cdot \mathrm{kg}^{-1}$, respectively). Supplemental doses of this anaesthetic mixture, $5 \%$ of the initial dose, were given if needed to maintain surgical anaesthesia. The cats were placed in the supine position on an operating table and breathed spontaneously.

\section{Measurements}

Catheters were inserted into the left femoral artery and vein for monitoring systemic blood pressure $\left(P_{\text {sys }}\right)$ and for intravenous administration of fluids. $P$ sys was measured with a differential pressure transducer Statham). The trachea was exposed in the neck and a cannula (7 mm O.D., $40 \mathrm{~mm}$ long) was inserted just below the cricoid cartilage. The tracheal cannula was connected to a flow pressure transducer (Fleisch type 0) of a pneumotachograph (Gould) for measuring airflow $\left(V^{r}\right)$. The other end of the Fleisch head was connected to a main tube by means of a T-piece. $P$ tr was measured with a differential pressure transducer (Statham). $P$ tr - relative to ambient pressure - was obtained from a side arm of the T-piece.
The breathing circuit has been described in detail previously [9]. Briefly, a constant bias flow of about $18 \mathrm{~L} \cdot \mathrm{min}^{-1}$ was maintained in the main tube, which was sufficient to prevent rebreathing of expired air. By turning a 3-way tap, the direct connection of this tube to room air was occluded and the bias flow was then directed to an adjustable flow resistance. At the same time, the connection to a water manometer was accomplished. With the help of this manometer and the adjustable flow resistance a certain value of $P$ tr was preset. During an experimental run, a preset positive $P$ tr could then be established almost instantaneously by rapidly turning the tap. By reversing the bias flow, a preset negative $P$ tr could be applied. A large bottle filled with air $(50 \mathrm{~L})$ and a water manometer with wide tubes $(9 \mathrm{~cm}$ I.D.) were used to minimize pressure fluctuations in the main tube due to breathing by the cat.

Conductance of vagal afferent activity can be inhibited and finally blocked by cooling both cervical vagus nerves. The vagi were exposed in the mid-neck, freed from the carotid sheaths, and placed in the gold coated grooves of two cooling devices. The grooves were $13 \mathrm{~mm}$ long, $2 \mathrm{~mm}$ deep and $1 \mathrm{~mm}$ wide. A Peltier element (cooling) and a transistor (heating) were used to achieve the required temperature. The temperature of the vagi $\left(T_{\mathrm{vg}}\right)$ could be adjusted between 37 and $0^{\circ} \mathrm{C}$ $\left( \pm 0.1^{\circ} \mathrm{C}\right)$. Both nerves were immersed in oxygenated paraffin oil.

At the end of the experimental protocol, bilateral section of the cervical vagi was performed to eliminate all input from vagal lung receptors to central respiratory centres. Temperature of both proximal ends of the cut vagi were then maintained at $0^{\circ} \mathrm{C}$ to prevent sponta- neous excitations.

Hooked needle electrodes were used to obtain EMGs from inspiratory muscles. After a small abdominal incision had been made just below the ribcage, a pair of electrodes was inserted into the costal part of the diaphragm. A second pair of electrodes was inserted parasternally into the intercostal muscles approximately $1 \mathrm{~cm}$ lateral to the sternum in the third or fourth intercostal space. The electrical activity of the diaphragm and of the ICMs were amplified, filtered (150-3,000 Hz), rectified, and fed into a leaky integrator with a time constant of $50 \mathrm{~ms}$ (Neurolog, Digitimer). The partial pressure of carbon dioxide in end-tidal gas $\left(P \mathrm{ET}, \mathrm{CO}_{2}\right)$ was measured by a capnograph (Nellcor N-1000). Body temperature was taken from the rectum and maintained between $36-38^{\circ} \mathrm{C}$ by heating the operating table.

Signals representing integrated EMG activity of the diaphragm (EMGd) and of ICMs (EMGicms), $V, P$ tr, $P$ ET, $\mathrm{CO}_{2}, P_{\text {sys }}$, rectal temperature and $T_{\text {vg }}$ of both vagi were monitored continuously, and were sampled $(50 \mathrm{~Hz})$ with a computer (Compaq 386) and stored on hard disk for offline analysis.

\section{Experimental protocol}

Two different experimental protocols were used to study histamine-induced ETIA and CNAP-induced ETIA. 
Protocol 1. Histamine-diphosphate ( $300 \mu \mathrm{g})$ was administered intravenously after 10 control breaths had been recorded. After ETIA in response to histamine was apparent for at least eight breaths, $P$ tr was increased to $0.9 \mathrm{kPa}$ (CPAP) during an inspiration. CPAP was maintained at the adjusted level for at least five breaths, after which normal $P$ tr was restored during an expiration. In this way, we subsequently recorded: 1) breaths during control conditions; 2) breaths during the occurrence of histamine-induced ETIA; 3) breaths during application of CPAP; and 4) breaths at normal $P$ tr. Between two consecutive experiments, a recovery was allowed until blood pressure had returned to the level prior to the administration of histamine. ETIA disappeared much earlier.

Protocol 2. After 10 control breaths had been recorded at normal $P$ tr, airway pressure was decreased to $-0.9 \mathrm{kPa}$ during an expiration (CNAP), maintained at the adjusted level for at least 10 breaths and restored again to control conditions during an inspiration.

The two different protocols were carried out at the following values of $T_{\mathrm{vg}}$ : $37,22,14,12,10,8,6$ and $4^{\circ} \mathrm{C}$. In practice, Protocol 2 was carried out first and then Protocol 1. After completion of these experiments, the cervical vagi were transected, whereupon the two types of experiments were repeated. In five animals, we studied the effects of stimulation of the C-fibre endings on ETIA. Capsaicin $\left(50 \mu \mathrm{g} \cdot \mathrm{kg}^{-1}\right)$, a chemical known to activate $\mathrm{C}$-fibre endings in cats $[11,15]$, was administered by intravenous injection at three values of $T_{\mathrm{vg}}(37,10$ and $\left.4^{\circ} \mathrm{C}\right)$.

\section{Data analysis}

From the recordings, we determined end-tidal EMG activity and peak inspiratory EMG activity both for the diaphragm and ICMs. In order to eliminate the effects of instrumental, interanimal and intra-animal scatter, we quantified ETIA of each breath as the ratio of end-tidal EMG activity and mean peak inspiratory EMG activity obtained from at least five control breaths from the beginning of the same recording (EMGPK,CTRL), in formula:

\section{$\mathrm{ETIA}=($ end-tidal EMG activity $/ \overline{\mathrm{EMG}} \mathrm{PK}, \mathrm{CTRL}) \times 100 \%$}

Thus, end-tidal EMG activity was expressed relative to the mean peak inspiratory EMG activity of control breaths.

For both the diaphragm and ICMs, three mean values for ETIA were calculated from the results of at least five breaths during control conditions, during histamineinduced ETIA, and during CPAP, respectively (Protocol 1). Similarly, mean ETIA values were calculated for control breaths and CNAP (Protocol 2). Furthermore, the duration of expiration ( $t \mathrm{E}$ ) was calculated for all different types of experimental conditions: control, histamine, histamine+CPAP and CNAP.

End-tidal inspiratory activity may, in part, be due to incomplete decay of inspiratory activity during expiration. To evaluate this contribution, the measured inspiratory activity during the expiratory phase of the breathing cycle was fitted with the function $\mathrm{Ae}^{-t / \tau}+\mathrm{B}$, where $t$ is time, $\mathrm{A}$ and $\mathrm{B}$ are amplitudes, and $\tau$ is the time constant of the decay of inspiratory activity. The results for A, B and $\tau$, thus, followed from the fitting procedure. From a mathematical point of view, the end-tidal value of the exponential decay of inspiratory activity at time $t=t \mathrm{E}$ is fully determined by $\mathrm{Ae}^{-t E / \tau}$ As for ETIA, the contribution of the incomplete decay of inspiratory activity during expiration is presented as percentage of EMGPK, CTRL. The fitting procedure was applied to the early part of expiration in which the decay of inspiratory activity is most pronounced and, therefore, also best defined. This procedure was further applied to all breaths used for the determination of ETIA.

All data presented are means \pm standard error (SEM). Differences were evaluated for statistical significance using the Wilcoxon test for paired observations. A p-value of less than 0.05 was considered to be significant.

\section{Results}

In 8 out of 18 animals, ETIA was evoked in response to i.v. administration of $300 \mu \mathrm{g}$ of histamine at normal or at decreased $T_{\mathrm{vg}}$. In these eight animals, ETIA could also be induced by CNAP. In 9 out of the 18 animals, no ETIA was evoked by CNAP $(\geq-0.9 \mathrm{kPa})$. In the present study, the results of seven responders to both stimuli, histamine and CNAP, will be evaluated (in one responder no CPAP was applied). Mean body weight ( \pm SEM) of the responders $(5.1 \pm 0.3 \mathrm{~kg} ; \mathrm{n}=8)$ was not significantly different from that of the nonresponders $(4.7 \pm 0.2 \mathrm{~kg}$; $\mathrm{n}=10$ ).

Original recordings of integrated EMG activity obtained from inspiratory muscles before and after i.v. administration of histamine and during subsequent application of CPAP are shown in figure 1. This figure shows that the increases in the end-tidal levels of integrated EMGDI and EMGICMs activities (trace 1 and 2, respectively) start about $14 \mathrm{~s}$ after the administration of histamine. Immediately after the application of CPAP, indicated by the sharp increase of $P$ tr (trace 3 ), the end-tidal levels of both EMG activities return to their baseline levels. This figure further demonstrates that after CPAP is released the end-tidal levels of EMG activities are increased again. Thus, ETIA was increased by histamine and the histamine-induced ETIA was suppressed by CPAP. The decrease of $P_{\text {sys }}$ in response to the administration of histamine is, shown in trace 5.

In response to intravenous injection of $300 \mu \mathrm{g}$ of histamine, mean arterial blood pressure $\left(\bar{P}_{\text {sys }}\right)$ decreased significantly $(\mathrm{p}<0.05)$ compared to control in each animal and at all values of $T_{\mathrm{vg}}$. The average decrease in $\bar{P}_{\text {sys }}$ for all individual experiments together was $8.5 \pm 0.36 \mathrm{kPa}$ $(\mathrm{n}=56)$.

\section{Effects of vagal cooling on ETIA (fig. 2a)}

The mean results of histamine-induced ETIA in the diaphragm and ICMs obtained from the responders at 


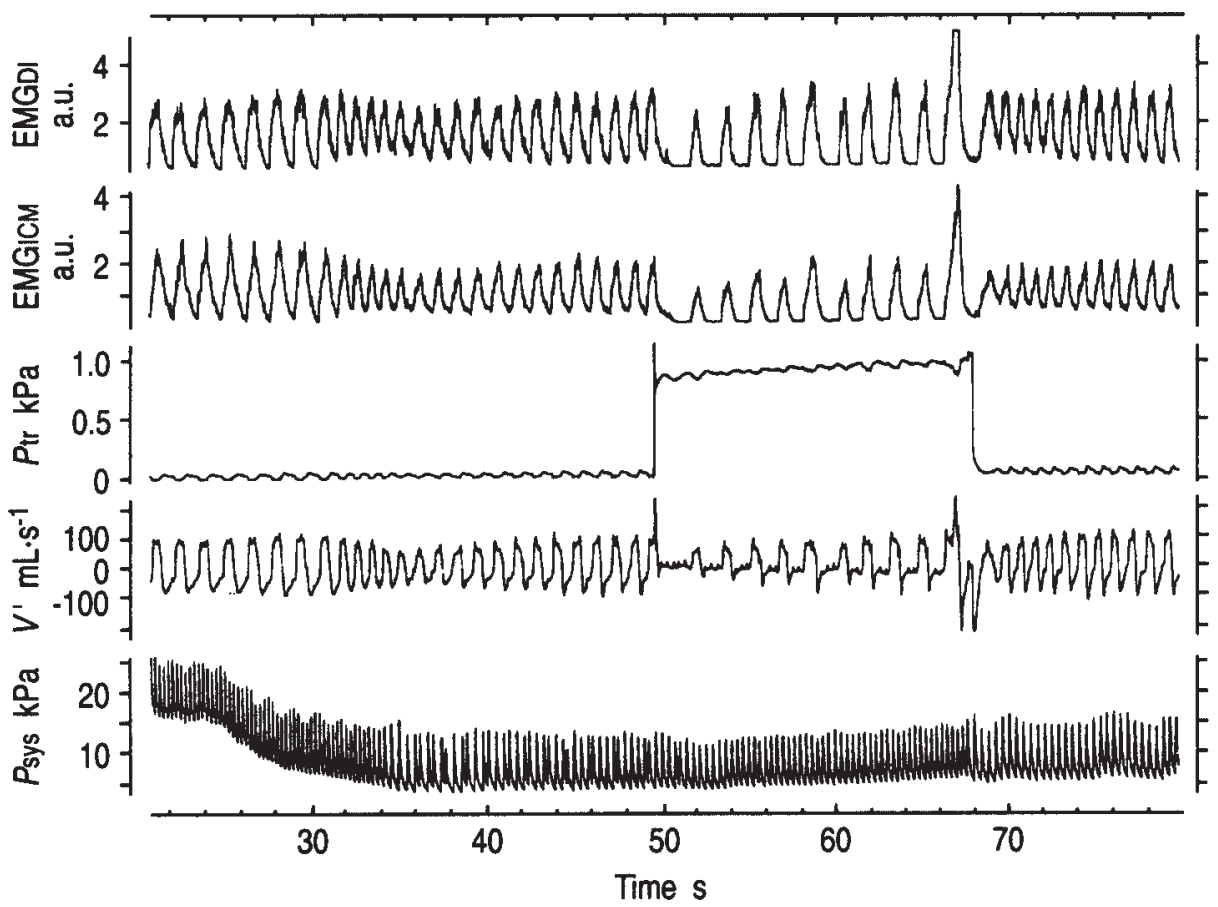

Fig. 1. - Recordings of integrated electromyographic (EMG) activity of the diaphragm EMGDI, integrated EMG activity of intercostal muscles (EMGICM), tracheal pressure (Ptr), airflow $\left(V^{\prime}\right)$ and systemic arterial blood pressure $(P$ sys $)$ after administration of histamine and during simultaneous application of continuous positive airway pressure (CPAP) for one representative animal. Histamine was administered at time $=14 \mathrm{~s}$ (not shown). Recording of $P$ tr shows when CPAP was applied. Note the onset of end-tidal inspiratory activity (ETIA) about $18 \mathrm{~s}$ after histamine was administered, and its suppression when CPAP was applied (trace 1 and 2). Temperature of the cervical vagi was $37^{\circ} \mathrm{C}$. a.u.: arbitrary units.
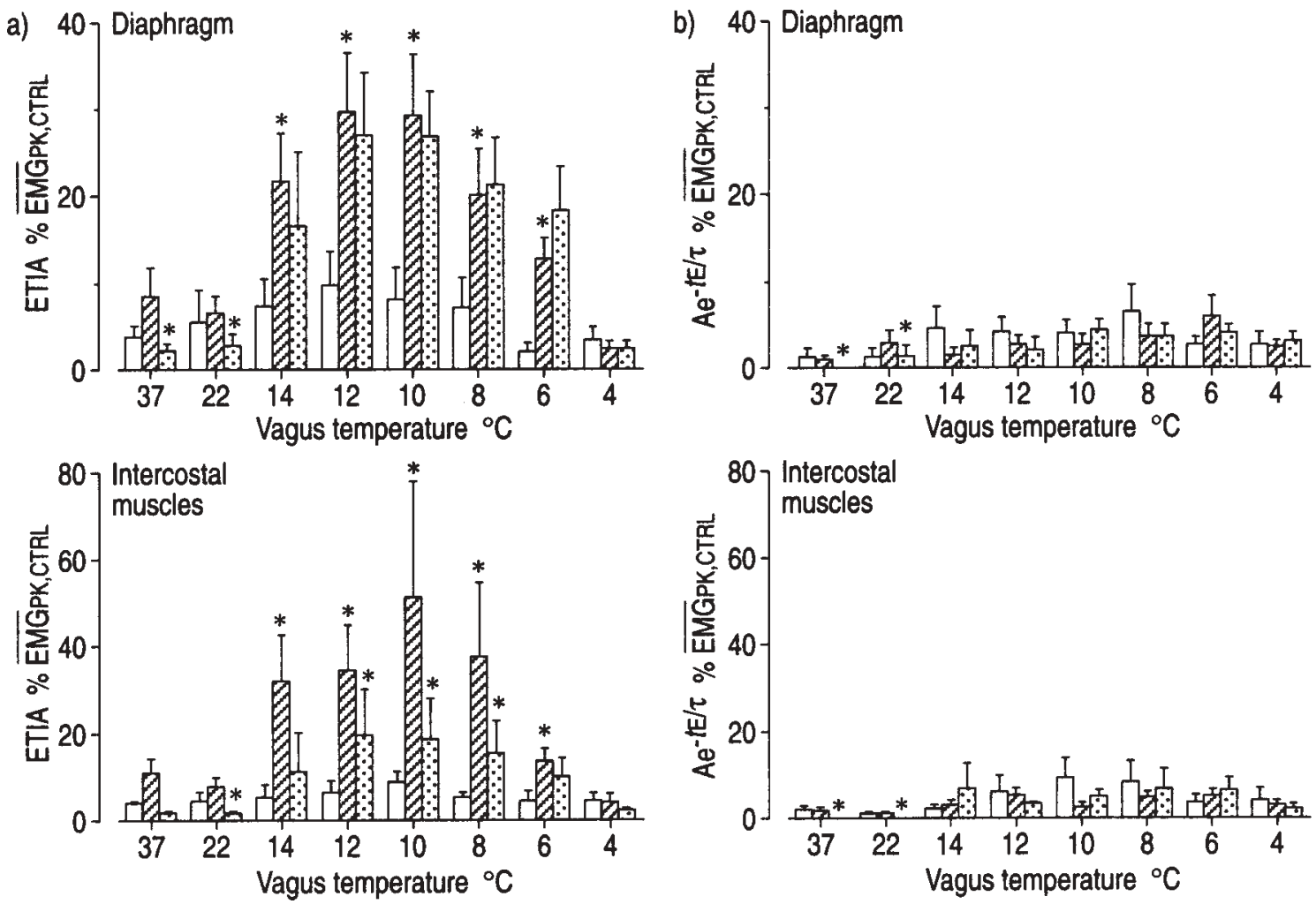

Fig. 2. - a) End-tidal inspiratory activity (ETIA) obtained from seven cats during control, after administration of histamine, and during subsequent CPAP (0.9 kPa) at different vagal temperatures. EMGPK,CTRL: mean peak electromyographic (EMG) activity obtained from control breaths. Asterisks indicate statistically significant differences in ETIA between histamine and control values, and between continuous positive airway pressure (CPAP) and histamine values for that temperature (*: $\mathrm{p}<0.05$; Wilcoxon-test for paired observations). b) Estimated end-tidal values (A: amplitude; $\tau$ : time constant; $t \mathrm{E}$ : duration of expiration) of the decaying inspiratory EMG activity during expiration. Data are presented as mean \pm SEM. $\square:$ control; $\nabla / \lambda$ : histamine; $\because$ : CPAP. 
different $T_{\mathrm{vg}}$ (ranging $37-4^{\circ} \mathrm{C}$ ) are shown in figure $2 \mathrm{a}$. As compared to the values at $T_{\mathrm{vg}}=37^{\circ} \mathrm{C}$, histamineinduced ETIA reaches much higher levels for $T_{\mathrm{vg}}$ between 14 and $6^{\circ} \mathrm{C}$ and for $T_{\mathrm{vg}}$ between 14 and $8^{\circ} \mathrm{C}$ for the diaphragm and ICMs, respectively.

At $T_{\mathrm{vg}}=37$ and $22^{\circ} \mathrm{C}$, histamine-induced ETIA in the diaphragm is significantly inhibited by CPAP. In ICMs the difference between the mean values (histamine versus histamine+CPAP) just failed to reach significance $(\mathrm{p}=0.07)$ at $T_{\mathrm{vg}}=37^{\circ} \mathrm{C}$, but, as with the diaphragm, this difference is significant at $T_{\mathrm{vg}}=22^{\circ} \mathrm{C}$. In the diaphragm, CPAP no longer suppressed histamine-induced ETIA at $T_{\mathrm{vg}}$ between 14 and $6^{\circ} \mathrm{C}$. In 4 out of 7 cats, histamine-induced ETIA in the diaphragm even increased in response to CPAP at $T_{\mathrm{vg}}=12^{\circ} \mathrm{C}$.

At $T_{\mathrm{vg}}$ between 12 and $8^{\circ} \mathrm{C}$, ETIA values in ICMs were significantly lower during CPAP than after histamine alone, but remained higher than control levels, which is in contrast to the findings at $T_{\mathrm{vg}}=37$ and $22^{\circ} \mathrm{C}$ where ETIA after CPAP was smaller than ETIA in control conditions. Thus, as compared to the suppressing influence of CPAP on histamine-induced ETIA at $T_{\mathrm{vg}}=37$ and $22^{\circ} \mathrm{C}$, this influence is relatively smaller at $T_{\mathrm{vg}} \leq 14^{\circ} \mathrm{C}$. Between $T_{\mathrm{vg}}=8$ and $4^{\circ} \mathrm{C}$ ETIA declines sharply, and for $T_{\mathrm{vg}}=4^{\circ} \mathrm{C}$ no change in ETIA is found in response to administration of histamine. At $T_{\mathrm{vg}}=4^{\circ} \mathrm{C}$ conduction in the afferents of both SARs and RARs is known to be largely blocked $[17,18]$.

\section{Negative airway pressure (fig.3a)}

In all animals in which ETIA could be evoked by histamine, ETIA was also induced by CNAP ( $P$ tr $=-0.9$ $\mathrm{kPa}$ ). ETIA values in response to CNAP were larger for the ICMs than for the diaphragm (fig. 3a). At $T_{\mathrm{vg}}=4^{\circ} \mathrm{C}$ no significant increase in ETIA was found in ICMs. However, in the diaphragm a small significant increase in ETIA remained.

At $T_{\mathrm{vg}}=37^{\circ} \mathrm{C}$ the magnitude of CNAP-induced ETIA is much larger than that of histamine-induced ETIA both for the diaphragm and the ICMs (figs. 2a and 3a), whereas at temperatures between 14 and $8^{\circ} \mathrm{C}$ ETIA values are comparable. Figure $3 \mathrm{a}$ further shows that ETIA values in response to CNAP are about the same for $T_{\mathrm{vg}}$ between 37 and $8^{\circ} \mathrm{C}$. Between $T_{\mathrm{vg}}=8$ and $4^{\circ} \mathrm{C}$, however, ETIA declines sharply (fig. 3a). This behaviour is similar to that seen for ETIA after histamine (fig. 2a).

\section{Capsaicin}

C-fibre stimulation by intravenous injection of capsaicin was performed in five cats, of which two were responders to both stimuli, histamine and CNAP. The administration of this drug caused the well-known respiratory effects, a short period of apnoea followed by rapid breathing (fig. 4). In each cat, capsaicin was injected at three different values for $T_{\mathrm{vg}}\left(37,10\right.$ and $\left.4^{\circ} \mathrm{C}\right)$, but in no single experiment was ETIA evoked.
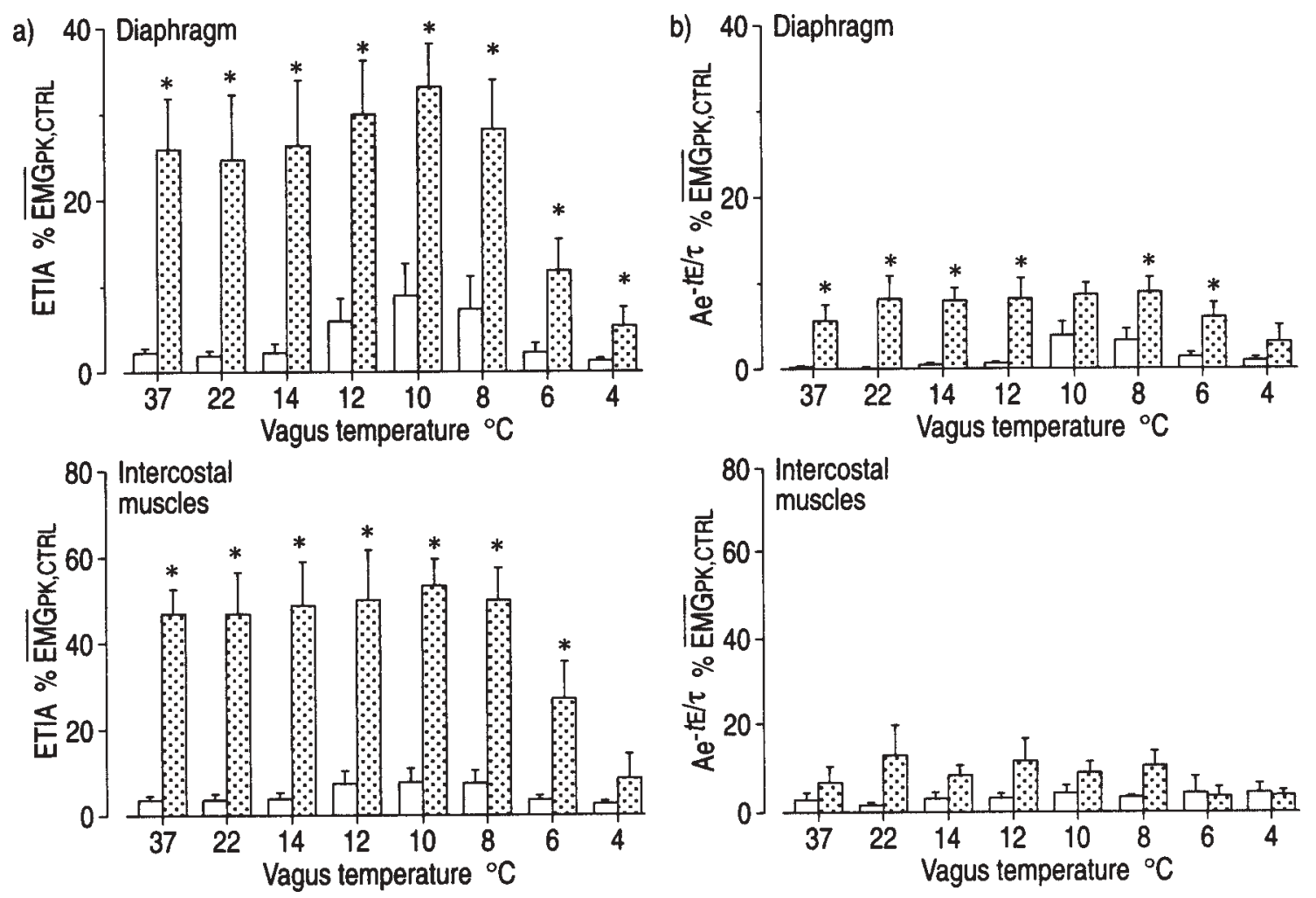

Fig. 3. - a) End-tidal inspiratory activity (ETIA) obtained from seven cats during control and during continuous negative airway pressure (CNAP) (-0.9 kPa) at different vagal temperatures. Asterisks indicate statistically significant differences between ETIA during CNAP and the corresponding control values for each temperature (*: p<0.05; Wilcoxon-test for paired observations). Note that ETIA values for the diaphragm are smaller than those for the intercostal muscles. b) Estimated end-tidal values of the decaying inspiratory electromyographic (EMG) activity during expiration. Data are presented as mean \pm SEM. $\square$ : control; $\because:$ : CNAP. For further abbreviations and explanation see legend to fig. 2. 


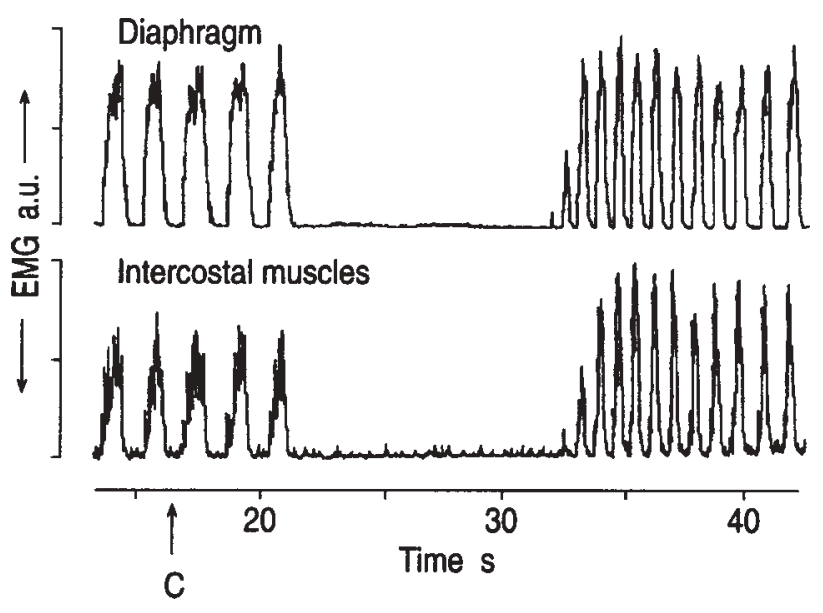

Fig. 4. - Recordings of integrated electromyographic (EMG) activity of the diaphragm and intercostal muscles (ICMs) before and after intravenous administration of capsaicin (C) (indicated by the arrow) for one representative animal. Note the apnoea and the subsequent increase in breathing frequency in response to capsaicin and the absence of end-tidal inspiratory activity. a.u.: arbitrary units.

\section{Vagotomy}

After both cervical vagus nerves had been cut, end-tidal EMG levels corresponded in all experiments to the electrical zero level. Hence, in neither condition - during control, after administration of histamine or during CNAP - was ETIA found after vagotomy.
ETIA and exponential decay of inspiratory activity during expiration (figs. $2 b$ and $3 b$ )

The contribution of the incomplete decay of inspiratory activity during expiration to ETIA was estimated from $\mathrm{Ae}^{-t E / \tau}$. The amplitude $\mathrm{A}$ and the time constant $\tau$ were obtained by fitting the measured inspiratory activity during expiration with the function $\mathrm{Ae}^{-t / \tau}+\mathrm{B}$ (see Methods). The results for $A^{-t E / \tau}$ are shown in figure $2 b$ (control, histamine, histamine+CPAP) and in figure $3 \mathrm{~b}$ (control, CNAP). As for ETIA, Ae ${ }^{-t E / \tau}$ was expressed as percentage of EMGPK,CTRL which facilitates the comparison with ETIA. In general, $\mathrm{Ae}^{-t E / \tau}$ is much smaller than ETIA both for the diaphragm and ICMs when ETIA is enhanced by histamine, histamine+CPAP or CNAP (figs. $2 \mathrm{~b}$ and $3 \mathrm{~b}$ ). This, however, is not true for control conditions where at several values of $T_{\mathrm{vg}} \mathrm{Ae}^{-t E / \tau}$ was more than half the value of ETIA for either the diaphragm or ICMs, or both. This especially occurs at lower vagal temperatures. After administration of histamine and for $T_{\mathrm{vg}}=14-8^{\circ} \mathrm{C}$ the average values for $\mathrm{Ae}^{-t E / \tau}$ for both the diaphragm and ICMs were about $12 \%$ of the corresponding values for ETIA. Similarly, at CNAP and for $T_{\mathrm{vg}}=37-8^{\circ} \mathrm{C}$ this percentage was about $23 \%$. Thus, in conditions in which ETIA is small, e.g. for $T_{\mathrm{vg}}=4^{\circ} \mathrm{C}$, most of the ETIA is due to the incomplete decay of inspiratory activity at the end of expiration. In contrast, in conditions in which ETIA is much elevated, ETIA is due mainly to the remaining determinants of ETIA, which will be discussed in the next section.

Table 1. - Duration of expiration $(t E)$ and time constant $(\tau)$ obtained from the decay of the inspiratory electromyographic (EMG) activity during expiration for three conditions: control; histamine+continuous positive airway pressure (CPAP)

Vagus temperature ${ }^{\circ} \mathrm{C}$

\begin{tabular}{|c|c|c|c|c|c|c|c|c|}
\hline & 37 & 22 & 14 & 12 & 10 & 8 & 6 & 4 \\
\hline \multirow[t]{2}{*}{$t \mathrm{E}$-control $\mathrm{s}$} & 0.92 & 0.94 & 0.92 & 0.97 & 1.08 & 1.13 & 1.27 & 1.33 \\
\hline & \pm 0.11 & \pm 0.11 & \pm 0.11 & \pm 0.12 & \pm 0.14 & \pm 0.13 & \pm 0.13 & \pm 0.10 \\
\hline \multirow[t]{2}{*}{$t \mathrm{E}$-histamine $\mathrm{s}$} & $0.71^{*}$ & $0.72 *$ & $0.66^{*}$ & $0.72 *$ & $0.88^{*}$ & 0.93 & $1.03^{*}$ & 1.23 \\
\hline & \pm 0.11 & \pm 0.09 & \pm 0.09 & \pm 0.08 & \pm 0.11 & \pm 0.11 & \pm 0.09 & \pm 0.14 \\
\hline \multirow[t]{2}{*}{$t \mathrm{E}-\mathrm{CPAP} \mathrm{s}$} & $1.37^{*}$ & $1.37 *$ & $1.00^{*}$ & $0.90^{*}$ & 1.04 & 1.08 & 1.03 & 1.14 \\
\hline & \pm 0.16 & \pm 0.14 & \pm 0.11 & \pm 0.08 & \pm 0.08 & \pm 0.10 & \pm 0.12 & \pm 0.10 \\
\hline \multicolumn{9}{|l|}{ Diaphragm } \\
\hline \multirow[t]{2}{*}{$\tau$-control s } & 0.18 & 0.17 & 0.27 & 0.31 & 0.32 & 0.40 & 0.34 & 0.28 \\
\hline & \pm 0.03 & \pm 0.04 & \pm 0.06 & \pm 0.07 & \pm 0.06 & \pm 0.19 & \pm 0.06 & \pm 0.05 \\
\hline \multirow[t]{2}{*}{$\tau$-histamine $\mathbf{s}$} & $0.14 *$ & 0.18 & 0.21 & $0.17^{*}$ & 0.24 & 0.29 & 0.33 & 0.28 \\
\hline & \pm 0.02 & \pm 0.04 & \pm 0.06 & \pm 0.03 & \pm 0.02 & \pm 0.05 & \pm 0.05 & \pm 0.04 \\
\hline \multirow[t]{2}{*}{$\tau$-CPAP s } & 0.12 & 0.17 & 0.21 & 0.22 & 0.32 & 0.28 & 0.28 & 0.26 \\
\hline & \pm 0.02 & \pm 0.07 & \pm 0.06 & \pm 0.04 & \pm 0.04 & \pm 0.02 & \pm 0.03 & \pm 0.02 \\
\hline \multicolumn{9}{|c|}{ Intercostal muscles } \\
\hline \multirow[t]{2}{*}{$\tau$-control $\mathrm{s}$} & 0.25 & 0.20 & 0.24 & 0.32 & 0.48 & 0.44 & 0.36 & 0.35 \\
\hline & \pm 0.05 & \pm 0.04 & \pm 0.04 & \pm 0.06 & \pm 0.10 & \pm 0.06 & \pm 0.08 & \pm 0.09 \\
\hline \multirow[t]{2}{*}{$\tau$-histamine $\mathrm{s}$} & 0.15 & 0.16 & 0.19 & 0.25 & 0.27 & 0.30 & 0.33 & 0.32 \\
\hline & \pm 0.02 & \pm 0.02 & \pm 0.02 & \pm 0.02 & \pm 0.03 & \pm 0.04 & \pm 0.03 & \pm 0.03 \\
\hline \multirow[t]{2}{*}{$\tau$-CPAP s } & 0.13 & 0.13 & 0.23 & 0.37 & 0.32 & 0.33 & 0.28 & 0.23 \\
\hline & \pm 0.02 & \pm 0.02 & \pm 0.04 & \pm 0.16 & \pm 0.06 & \pm 0.05 & \pm 0.03 & \pm 0.03 \\
\hline
\end{tabular}

Data are presented as mean \pm SEM for seven animals. Values after administration of histamine were compared with control, values during CPAP were compared with values after histamine. Wilcoxon matched-pairs signed-ranks test; $\mathrm{p}<0.05$ was considered significant. s: seconds; $*$ : $\mathrm{p}<0.05$. 
Table 2. - Duration of expiration ( $t E)$ and time constant $(\tau)$ obtained from the decay of the inspiratory electromyographic (EMG) activity during expiration for two conditions: control and continuous negative airway pressure (CNAP)

Vagus temperature ${ }^{\circ} \mathrm{C}$

\begin{tabular}{lccccccrr} 
& 37 & 22 & 14 & 12 & 10 & 8 & \multicolumn{1}{c}{6} & 4 \\
\hline$t$ E-controls s s & 0.99 & 0.99 & 0.97 & 0.93 & 0.87 & 0.98 & 1.19 & 1.29 \\
& \pm 0.13 & \pm 0.12 & \pm 0.13 & \pm 0.15 & \pm 0.14 & \pm 0.15 & \pm 0.11 & \pm 0.11 \\
$t$ E-CNAP s & $0.64^{*}$ & $0.52^{*}$ & $0.49^{*}$ & $0.47^{*}$ & $0.48^{*}$ & $0.53^{*}$ & 0.85 & 1.20 \\
& \pm 0.20 & \pm 0.13 & \pm 0.12 & \pm 0.13 & \pm 0.11 & \pm 0.14 & \pm 0.17 & \pm 0.17
\end{tabular}

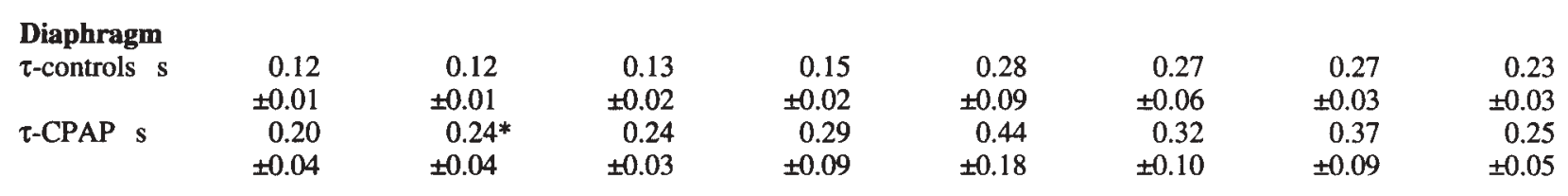

\begin{tabular}{|c|c|c|c|c|c|c|c|c|}
\hline \multicolumn{9}{|c|}{ Intercostal muscles } \\
\hline$\tau$-controls $\mathrm{s}$ & 0.22 & 0.21 & 0.25 & $\begin{array}{r}0.27 \\
+0.08\end{array}$ & 0.28 & 0.39 & 0.41 & 0.37 \\
\hline$\tau$-CPAP S & $\begin{array}{r} \pm 0.06 \\
0.24\end{array}$ & $\begin{array}{r} \pm 0.05 \\
0.35\end{array}$ & $\begin{array}{r} \pm 0.06 \\
0.27\end{array}$ & $\begin{array}{r} \pm 0.08 \\
0.39\end{array}$ & $\stackrel{ \pm 0.06}{0.34 *}$ & $\begin{array}{c} \pm 0.12 \\
0.37 *\end{array}$ & $\begin{array}{r} \pm 0.10 \\
0.29\end{array}$ & $\begin{array}{r} \pm 0.10 \\
0.32\end{array}$ \\
\hline & \pm 0.05 & \pm 0.07 & \pm 0.03 & \pm 0.11 & \pm 0.07 & \pm 0.10 & \pm 0.03 & \pm 0.05 \\
\hline
\end{tabular}

Data are presented as mean \pm SEM for seven animals. Values during continuous negative airway pressure (CNAP) were compared with control. Wilcoxon matched-pairs signed-ranks test; $\mathrm{p}<0.05$ was considered significant. *: $\mathrm{p}<0.05$.

The duration of expiration, $t \mathrm{E}$, and the time constant, $\tau$, for the decay of inspiratory activity during expiration are important determinants of $\mathrm{Ae}^{-t E / \tau}$. The results for $t \mathrm{E}$ and $\tau$ are shown in tables 1 and 2 . These data show that, in general, $t \mathrm{E}$ and $\tau$ vary with $T_{\mathrm{vg}}$ and with changing experimental conditions.

\section{Discussion}

ETIA was induced in response to CNAP or intravenous injection of histamine both in the feline diaphragm and ICMs. At $T_{\mathrm{vg}}$ between 8 and $4^{\circ} \mathrm{C}$, the effects of both stimuli on ETIA decreased to low levels with decreasing temperature. After vagotomy, histamine and CNAP could no longer evoke ETIA. At $T \mathrm{vg}=37^{\circ} \mathrm{C}$ histamine-induced ETIA was suppressed by CPAP, whereas at $T \mathrm{vg}$ between 14 and $8^{\circ} \mathrm{C}$ this suppression was diminished. These effects were most pronounced for the diaphragm. Both histamine-induced ETIA and CNAPinduced ETIA were larger in ICMs than in the diaphragm (figs. 2a and 3a).

After vagotomy, no ETIA was observed in response to CNAP, which demonstrates that ETIA is not evoked by extra vagal mechanisms. A considerable fraction of our experimental animals did not respond to histamine and CNAP. This shows that there is a wide natural variability in cats in terms of showing ETIA in response to the applied stimuli. In human subjects, a similar variability is found for the response of the airway resistance during histamine challenge tests.

ETIA has also been studied in other species. In rabbits, ETIA has been evoked in response to a decreased $P \operatorname{tr}$ [7] and after administration of histamine, both in the diaphragm and in ICMs [10]. In man, ETIA has been observed after administration of histamine [6].
ETIA and exponential decay of inspiratory activity during expiration

It is known from the literature $[15,19-21]$ that the activities of SARs and/or RARs are affected when changing the experimental conditions from control to CNAP or CPAP and after administration of histamine. In turn, the activities of these receptors affect the behaviour of the inspiratory off-switch and on-switch mechanisms, and, therefore, also $t$ E, EMGPK and $\tau$. This was also reflected by the changes in $t \mathrm{E}$ and $\tau$ that were observed in individual cats when changing from one experimental condition to another (tables 1 and 2). Increases in EMGPK or $t \mathrm{E} / \tau$ or both will result in a larger contribution of the incomplete decay of inspiratory activity to ETIA. The changes in these parameters induced by the stimuli applied imply that the contribution of the incomplete decay of inspiratory activity to ETIA during stimulation cannot be determined independently from, e.g. control breaths. For this reason, we had to determine this contribution and ETIA from the same EMGs, as described in Methods. Figures $2 b$ and $3 \mathrm{~b}$ show that the contribution of the incomplete decay of inspiratory activity to ETIA is modest in conditions in which ETIA is much elevated. It can be further inferred from figures 2 and 3 that the behaviour of ETIA as a function of $T \mathrm{vg}$ is not much affected by the contribution of the incomplete decay of inspiratory activity to ETIA. In other words, the initial rise in ETIA for histamine (and also for histamine $+\mathrm{CPAP}$ ) at $T \mathrm{vg}=14^{\circ} \mathrm{C}$ and its decline for $T \mathrm{vg}$ below $10^{\circ} \mathrm{C}$ can be safely attributed to changes in the remaining determinants of ETIA, and this also applies to the decline in ETIA for $T_{\mathrm{vg}}$ below $8^{\circ} \mathrm{C}$ for CNAP (fig. 3). A further detail of figure 3 is, that if the individual values for ETIA at $T_{\mathrm{vg}}=4^{\circ} \mathrm{C}$ are corrected for the corresponding values of 
$\mathrm{Ae}^{-t E / \tau}$ the mean value of corrected ETIA is still significantly different from control but very small (about $2 \%$ of EMGPK,CTRL).

\section{C-fibre endings and ETIA}

BADIER et al. [10] used procaine to selectively block the electric conduction of thin vagal fibres, and concluded that stimulation of thin sensory fibres elicits ETIA. However, differentiation between C-fibre endings and RARs (both have thin vagal afferents) was not possible. In the present study, we used vagal cooling to selectively block the conduction of the afferents from lung receptors. It has been shown that at temperatures below $5^{\circ} \mathrm{C}$ the conduction in myelinated fibres (from SARs and RARs) is almost completely blocked, whereas a large fraction of the activity of unmyelinated C-fibres is still transmitted $[17,18]$. At $T_{\mathrm{vg}}=4^{\circ} \mathrm{C}$ hardly any histamine-induced ETIA was found, indicating that C-fibre endings are not involved in inducing ETIA (fig. 2). We observed similar effects of vagal cooling on CNAP-induced ETIA (fig. 3). Our conclusion that $\mathrm{C}$-fibre endings are not involved in ETIA is further supported by our observation that chemical stimulation of C-fibres by capsaicin does not induce ETIA (fig. 4). It is not likely, therefore, that stimulation of C-fibre endings by histamine [15] has attributed to the genesis of ETIA in our experiments. After vagotomy, the different stimuli no longer evoked ETIA and, thus, the occurrence of ETIA depends on vagal reflex mechanisms. Since C-fibre endings appear to play no role, ETIA may be attributed to reflex effects of either RARs or SARs or both.

\section{SARs, RARs and ETIA}

It is generally accepted that stimulation of RARs excites inspiratory activity, whereas this activity can be inhibited by stimulation of SARs [15]. RAR activity increases and SAR activity decreases when Ptr is lowered [19-21]. Thus, CNAP-induced ETIA (fig. 3) may result from excitation of inspiratory motoneurons by RARs or lack of inhibition of these neurons by SARs or both. RARs and, in particular, SARs are stimulated by increased functional residual capacity (FRC) as induced by CPAP [15]. As a consequence, the nearly complete suppression of histamine-induced ETIA at $T_{\mathrm{vg}}=37^{\circ} \mathrm{C}$ (fig. 2a) in response to CPAP must be attributed to inhibition of inspiratory motoneurons activity by SARs. Lack of inhibition of inspiratory motoneurons by SARs, however, cannot be the only mechanism underlying ETIA because elimination of all afferent input from SARs by vagotomy does not result in ETIA. Hence, the primary cause for ETIA to occur in our experiments was the excitation of inspiratory motoneurons by RARs, where the amplitude of ETIA was modulated by SAR activity through inhibition of inspiratory activity. The indicated role for RARs was further supported by the fact that high levels of ETIA could be induced by administration of histamine, a drug which is known to stimulate RARs.

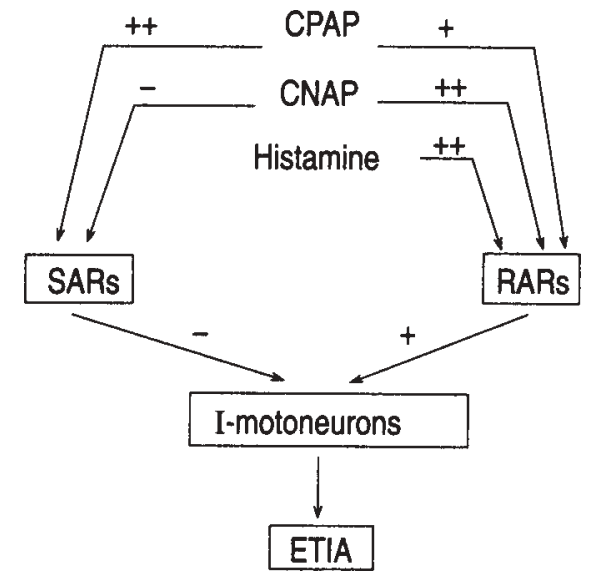

Fig. 5. - Schematic presentation of the effects of continuous positive airway pressure (CPAP), continuous negative airway pressure (CNAP) and histamine on slowly adapting receptor (SAR) and rapidly adapting receptor (RAR) activity, and of the impact of SAR and RAR activity on inspiratory (I) motoneuron activity and endtidal inspiratory activity (ETIA). Plus and minus signs refer to stimulation and inhibition, respectively.

The model resulting from the above discussion is depicted in figure 5. Further evidence for this model was obtained from the effects of vagal cooling. In dogs and cats, there is an overlap in conduction velocities of RARs and SARs, though the mean value for RARs appears to be lower [22]. In single fibre studies, it has been found that at vagal temperatures below $14^{\circ} \mathrm{C}$ conduction in afferents of SARs and RARs is progressively reduced [17, 18, 23]. However, diminished conduction in afferents of SARs occurs at slightly higher temperatures $\left(12-10^{\circ} \mathrm{C}\right)$ than in the afferents of RARs $\left(9-7^{\circ} \mathrm{C}\right)$ [17]. In the present study, several temperatures in the range of 37 down to $4^{\circ} \mathrm{C}$ were applied. This explains the general behaviour of histamine-induced ETIA with decreasing $T_{\mathrm{vg}}$, which first increases at $T_{\mathrm{vg}}=14^{\circ} \mathrm{C}$ (reduced central input of SAR activity) and then decreases again at temperatures below $10^{\circ} \mathrm{C}$ (reduced central input of RAR activity). For $T_{\mathrm{vg}}<14^{\circ} \mathrm{C}$, the percentage decrease in ETIA due to CPAP is substantially smaller than at $T_{\mathrm{vg}}=37^{\circ} \mathrm{C}$. This can be attributed to the reduced central input from SARs for $T_{\mathrm{vg}}<14^{\circ} \mathrm{C}$. At vagal temperatures where the conduction of SAR activity is (largely) blocked whilst that of most RARs is still transmitted, CPAP may augment ETIA through additional stimulation of RARs. This explains the augmentation of histamine-induced ETIA by CPAP which was seen in the diaphragm at $T_{\mathrm{vg}}=8-6^{\circ} \mathrm{C}$ in some of our experimental animals (fig. 2).

At $T_{\mathrm{vg}}=37^{\circ} \mathrm{C}$ the magnitude of ETIA in response to CNAP (-0.9 kPa) was larger than that in response to histamine both for the diaphragm and ICMs. When SAR activity was diminished by vagal cooling, ETIA values after histamine and during CNAP were of the same magnitude. This suggests that the magnitude of RAR activation is comparable for both types of stimuli. SAR activity is already reduced at $P \mathrm{tr}=-0.9 \mathrm{kPa}$ [19] and, therefore, at this $P$ tr the decrease in central input of SAR activity due to vagal cooling will be considerably less than the corresponding decrease at $P_{\mathrm{vg}}=0 \mathrm{kPa}$ (control). 
In other words, at $P \operatorname{tr}=-0.9 \mathrm{kPa}$, vagal cooling can hardly reduce afferent activity of SARs any further, resulting in much more constant levels of ETIA for $T \mathrm{vg}$ down to about $8^{\circ} \mathrm{C}$ (fig. 3) as compared to those found after histamine (fig. 2). Figures $2 \mathrm{a}$ and $3 \mathrm{a}$ are comparable for lower temperatures $\left(T \mathrm{vg}\right.$ from $8^{\circ} \mathrm{C}$ down to $4^{\circ} \mathrm{C}$ ) ETIA values decline to low levels. In this range, the activity in the afferents of RARs is diminished. The finding that at $T \mathrm{vg}=4^{\circ} \mathrm{C}$ there is still a small but significant increase in ETIA in response to CNAP in the diaphragm should be ascribed to the presence of some afferent activity from RARs. Whilst ETIA decreases by vagal cooling, FRC will decrease simultaneously. During CNAP, this additional decrease in FRC will result in enhanced mechanical stimulation of RARs. Such an enhanced stimulation of RARs may explain why ETIA at $T_{\mathrm{vg}}=4^{\circ} \mathrm{C}$ is still significantly different from control values in the diaphragm during CNAP, while this is not the case when induced by histamine (figs. 2 and 3). In other words, the diminishing effect of vagal cooling (below $8^{\circ} \mathrm{C}$ ) on the afferent activity of RARs during CNAP may, at least in part, be compensated for by the enhanced stimulation of RARs due to the concomitant decrease in FRC and end-tidal transpulmonary pressure [24].

\section{Origin of histamine-induced ETIA}

It has been shown, in animals, that the activity of RARs increases with decreasing $P \operatorname{tr}[11,21]$, by hyperinflation [11], and by administration (intravascular or inhalation) of histamine $[11,12,14,25,26]$. Histamine is believed to stimulate RARs both indirectly by a mechanical effect and by a direct chemical effect [12, 27]. Histamine-induced ETIA in cats may, therefore, have a dual origin arising from stimulation of RARs, by a direct, chemical effect and indirectly by a mechanical effect.

A difficulty in investigating reflex functions mediated by RARs is that all the naturally occurring chemicals known to stimulate RARs, such as histamine, are themselves powerful bronchoconstrictors, acting directly on airway smooth muscle and, thus, also stimulating RARs indirectly.

Although the stimuli (histamine and CNAP) are different in our experiments, the effects in terms of ETIA appear to be about the same at a vagus temperature of $10^{\circ} \mathrm{C}$ both for the diaphragm and ICMs (figs. 2 and 3). This indicates a comparable stimulation of RARs, since at this temperature most of the afferent activity from SARs will be abolished. CNAP is a purely mechanical stimulus exciting RARs. Although the present study was not designed to differentiate between chemical and mechanical stimulation of RARs by histamine, the above results for ETIA at $T \mathrm{vg}=10^{\circ} \mathrm{C}$ suggest that in histamineinduced ETIA mechanical stimulation of RARs might be the more important stimulus.

MULLER et al. [28] have shown that ETIA and hyperinflation develops in asthmatics when their bronchodilatory medication is withheld for $12 \mathrm{~h}$. These results can be explained by an increased stimulation of RARs due to rebound bronchoconstriction. We think that in asthma the balance between the levels of activity of RARs and SARs may be disturbed, resulting in ETIA (fig. 5). This is supported by the observation that CPAP reduced the load on inspiratory muscles in asthmatics after inhalation of histamine [29]. It is not yet clear whether the sensitivity of the receptors, the number of receptors, the central processing of afferent impulses, or a combination of these factors is involved in this balance.

In conclusion: 1) in spontaneously breathing cats, ETIA can be induced both in the diaphragm and ICMs by intravenous administration of histamine and by application of CNAP; 2) for these conditions, the incomplete decay of inspiratory activity during expiration constitutes only a modest part of ETIA; 3) ETIA is mediated by a vagal reflex; 4) C-fibre endings are not involved in ETIA; 5) stimulation of RARs excites ETIA whereas ETIA is inhibited by stimulation of SARs.

\section{References}

1. Woolcock AJ, Rebuck AS, Cade JF, Read J. Lung volume changes in asthma measured concurrently by two methods. Am Rev Respir Dis 1971; 104: 703-709.

2. Jiang TX, Deschepper K, Demedts M, Decramer M. Effects of acute hyperinflation on the mechanical effectiveness of the parasternal intercostals. Am Rev Respir Dis 1989; 139: 522-528.

3. Demedts M. Mechanisms and consequences of hyperinflation. Eur Respir J 1990; 3: 617-618.

4. Martin JG, Powell E, Shore SA, Emrich J, Engel LA. The role of respiratory muscles in the hyperinflation of bronchial asthma. Am Rev Respir Dis 1980; 121: 441-447.

5. Martin JG, Habib M, Engel LA. Inspiratory muscle activity during induced hyperinflation. Respir Physiol 1980; 39: 303-313.

6. Muller N, Bryan AC, Zamel N. Tonic inspiratory muscle activity as a cause of hyperinflation in histamine-induced asthma. J Appl Physiol: Respirat Environ Exercise Physiol 1980; 49: 869-874.

7. Patberg WR, Effect of graded vagal blockade and pulmonary volume on tonic inspiratory activity in rabbits. Pflügers Arch 1983; 398: 88-92.

8. van der Grinten CPM, de Vries WR, Luijendijk SCM. Vagally-mediated modification of inspiratory activity by changes in airway pressure. Respir Physiol 1992; 90: 159172.

9. Meessen NEL, van der Grinten CPM, Luijendijk SCM, Folgering HTM. Tonic activity in inspiratory muscles during continuous negative airway pressure. Respir Physiol 1993; 92: 151-166.

10. Badier M, Jammes Y, Romero-Colomer P, Lemerre C. Tonic activity in inspiratory muscles and phrenic motoneurons by stimulation of vagal afferents. J Appl Physiol 1989; 66: 1613-1619.

11. Armstrong DJ, Luck JC. A comparative study of irritant and type J receptors in the cat. Respir Physiol 1974; 21: 4760.

12. Vidruk EH, Hahn HL, Nadel JA, Sampson SR. Mechanisms by which histamine stimulates rapidly adapting receptors in dog lungs. J Appl Physiol: Respirat Environ Exercise Physiol 1977; 43: 397-402.

13. Matsumoto S. Effects of ammonia and histamine on lung irritant receptors in the rabbit. Respir Physiol 1989; 77: 301-308. 
14. Yu J, Roberts AM. Indirect effects of histamine on pulmonary rapidly adapting receptors in cats. Respir Physiol 1990; 79: 101-110.

15. Coleridge HM, Coleridge JCG. Reflexes evoked from tracheobronchial tree and lungs. In: Cherniack NS, Widdicombe JG, eds. Handbook of Physiology. The Respiratory System: Control of Breathing. Vol. II. Bethesda, MD, American Physiological Society, 1986; pp. 395-429.

16. Meessen NEL, van der Grinten CPM, Folgering HTM, Luijendijk SCM. Role of lung receptors in the generation of histamine-induced tonic inspiratory activity. Eur Respir J 1990; 3: 140s

17. Pisarri TE, Yu J, Coleridge HM, Coleridge JCG. Background activity in pulmonary vagal $\mathrm{C}$-fibers and its effects on breathing. Respir Physiol 1986; 64: 29-43.

18. Jonzon A, Pisarri TE, Roberts AM, Coleridge JCG, Coleridge HM. Attenuation of pulmonary afferent input by vagal cooling in dogs. Respir Physiol 1988; 72: 19-34.

19. Adrian ED. Afferent impulses in the vagus and their effect on respiration. J Physiol (Lond) 1933; 79: 332-358.

20. Sellick H, Widdicombe JG. The activity of lung irritant receptors during pneumothorax, hyperpnoea and pulmonary vascular congestion. J Physiol (Lond) 1969; 203: 359-381.

21. Sellick H, Widdicombe JG. Vagal deflation and inflation reflexes mediated by lung irritant receptors. Q J Exp Physiol 1970; 55: 153-163.
22. Sant'Ambrogio G. Information arising from the tracheobronchial tree of mammals. Physiol Rev 1982; 62: 531-569.

23. Widdicombe JG. Respiratory reflexes from the trachea and bronchi of the cat. J Physiol (Lond) 1954; 123: 55-70.

24. van der Grinten CPM. Vagal mechanisms in respiratory control. PhD thesis, State University Limburg, The Netherlands, Maastricht, Datawyse, 1991.

25. Sellick H, Widdicombe JG. Stimulation of lung irritant receptors by cigarette smoke, carbon dust, and histamine aerosol. J Appl Physiol: Respirat Environ Exercise Physiol 1971; 31: 15-19.

26. Winning AJ, Widdicombe JG. The effect of lung reflexes on the pattern of breathing in cats. Respir Physiol 1976; 27: 253266.

27. Snapper JR, Drazen JM, Loring SH, Braash PS, Ingram RH Jr. Vagal effects on histamine, carbachol, and prostaglandin $\mathrm{F}_{2 \alpha}$ responsiveness in the dog. J Appl Physiol: Respirat Environ Exercise Physiol 1979; 47: 13-16.

28. Muller N, Bryan AC, Zamel N. Tonic inspiratory muscle activity as a cause of hyperinflation in asthma. J Appl Physiol: Respirat Environ Exercise Physiol 1981; 50: 279-282.

29. Martin JG, Shore SA, Engel LA. Effect of continuous positive airway pressure on respiratory mechanics and pattern of breathing in induced asthma. Am Rev Respir Dis 1982; 126: 812-817. 